Iraqi Journal of Information and Communications Technology(IJICT)

Conference Series: The $1^{\text {st }}$ Conference of Applied Researches

in Information Engineering(ARIE2021), 2021

ISSN:2222-758X

e-ISSN:2789-7362

\title{
AN EFFICIENT ADAPTATION OF UHD VIDEO STREAMING OVER INTERNET
}

\author{
Hiba K. Abdulazeez ${ }^{1}$, Nasser N. Khamiss ${ }^{2}$ \\ ${ }^{1}$ Department of Information, Iraqi Geological Survey, Iraq \\ ${ }^{2}$ College of Information Engineering, Al-Nahrain University, Baghdad, Iraq \\ habok88@gmail.com ${ }^{1}$, nasser@coie-nahrain.edu.iq ${ }^{2}$ \\ Received:17/4/2021, Accepted:28/5/2021
}

\begin{abstract}
The main challenge of multimedia applications is how to transmission the Ultra-High Definition (UHD) video streaming in real-time over the internet. Real-time video streaming suffers from difficulties to be the flexible and efficient cause of the wide variation of the available internet bandwidth. To avoid the problems that introduce with the internet, in this work the HEVC with required video network adaptive streaming are proposed and tested using different six levels of three UHD video (4K, FHD, 720p, 4CIF, CIF and QCIF). Different experiments are applied to find the optimal configuration of $\mathbf{H . 2 6 5}$ encoding features for six levels to obtain the required PSNR with a range of 32-38 dB. The important part of this paper is a controller that worked incorporate with the encoder (H.265) to obtain the video streaming adaptation on the available bandwidth of the channel. The controller continuously reads the status of the channel buffer, then choosing the proper level of video to be transmitted over the channel. The work architecture is content two parts: First, the $\mathbf{H . 2 6 5}$ codec that applies on the three raw videos with optimal parameters configuration to compress them and get videos with lower bit rate and acceptable quality. Second, the compressed videos, based on controller selection, will be transmitted over two types of networks: Unicast and Multicast, and monitoring these two networks to evaluate their performance by obtaining different maps to choose the good one based on their effects on all levels of videos that are tested in this work.
\end{abstract}

keywords: UHD video, HEVC/H.265, RTP protocols, QoS, Unicast, Multicast.

\section{INTRODUCTION}

The increased use of video streaming with different resolutions for several kinds of terminal devices like mobile, tablet, PCs and High Definition TV (HDTV), and the diversity of services with the growing popularity of higher quality UHD video in these device applications are the main challenges of multimedia providers [1]. These challenges make the compression techniques with H.265/HEVC encoders is the way to get the better of the traffic bandwidth load balancing [2]. The main goal of this technique is to improve compression performance with good quality of bit-rate with a reduction of more than $50 \%$ from the previous standard. Moreover, H.265/HEVC have greater flexibility and more sophisticated features that can enable better compression and the possibility to store or transmit video more efficient than H.264/MPEG-4 AVC [3]. The purposes of this work are about video compression and streaming adaptation with the channel's bandwidth, where the internet is the media communication channel for a wide range of terminal devices. The main challenge is the available bandwidth where an unlimited number of users sharing it. The H.265 parameters that are directly affecting the bit rate and quality, like Quantization Parameter (QP), Constant Rate Factor (CRF), Group of Pictures, number of references (REF) and others are used to evaluate the optimal bit rate and quality for each level. The HEVC is used to produce the compressed video at each level. Based on the channel buffer status, the controller selects the proper level. While the reconstructed video, almost in the proper resolution, based on the user terminal device application. This technique has made video access to clients with high quality easily, also taking into account the channel status with technologies of the network that led the services of video streaming are available at any situation of network condition and any devices. This work also monitoring 
the wireless network parameters (packet loss, delay and jitter) that cause degradation in video quality [4]. This work will propose the optimal adaptation algorithm of video quality with variable bit-rate of real-time encoded video over the internet. The used streaming technique is based on the technique used in [5].

\section{A. (HEVC/H.265) Design and Feature}

The HEVC standard becomes the candidate to perform different goals, not just the compression efficiency but also it's designed to be used in several services, products and applications, also it is an integrated system that makes the data transporting easily and flexible of removing redundant data, as well as can execute by parallel processing architectures [6]. In this thesis, HEVC is used as the core part of the proposed encoder due to its properties. The block diagram of the HEVC encoder as given in the standard is described in Fig. 1 [7].

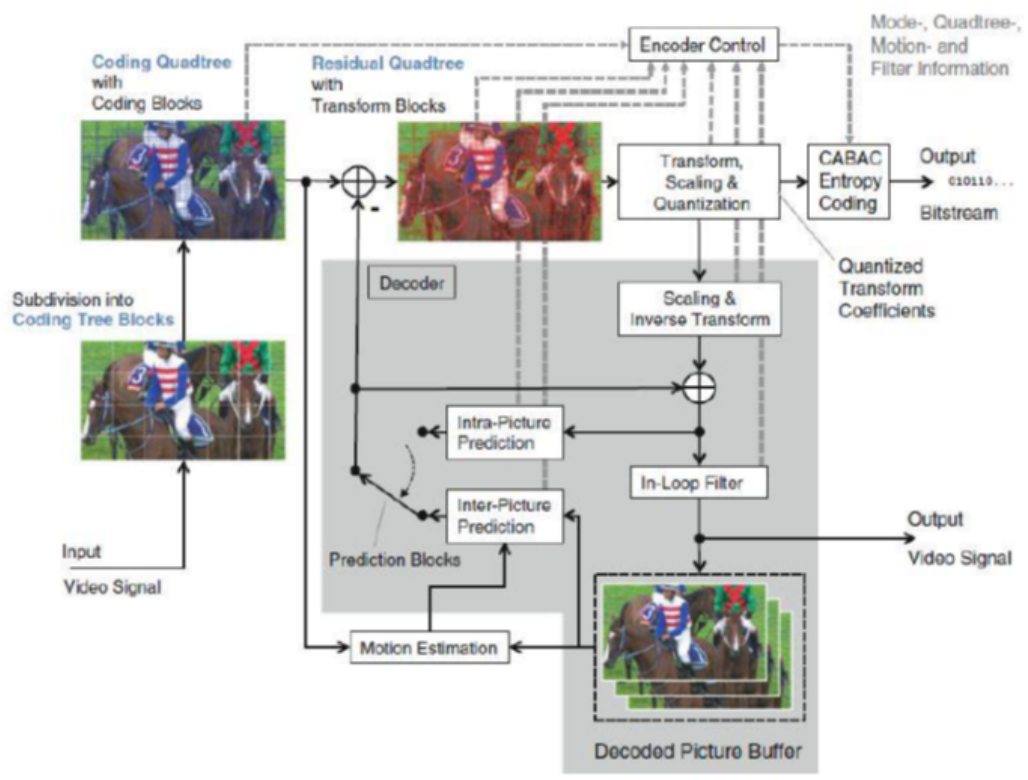

Figure 1: H.265 block diagram encoding [7]

The input frame was partitioned by using Coding Tree Unit (CTU) with sizes $8 \times 8,16 \times 16,32 \times 32,64 \times 64$ of macroblocks. The coefficients produced from the partitioning process are transmitted to the decoder after submitting into the quantization process. These techniques of macroblock partitioning can be used in the prediction process (intra and inter) to minimize data redundancy [8], Then the residual signal is produced by subtracting the prediction values from the original data, then applying the Discrete-Fourier Transform (DFT) algorithm to transform the spatial domain into the frequency domain to produce the transform coefficients that have been scaled [9]. After their steps applying the quantization process to lose inadequate information and then applying syntax-based context-adaptive binary arithmetic coder (SBAC) entropy coding [10] to get compressed bitstream and then transmit with the side information over the channel. The coefficients from transformed and quantized pass through Dequantization and inverse DFT to restore residuals then was summed with 
prediction values to obtain restored pixel values, and these values are used in intra-prediction with a current frame of video [11]. The results from these operations pass through the post-processing. Then the restored value and post-processed frame are saved into Decoded Picture Buffer (DPB) that is used for inter prediction of more frames [12]. The mechanism of video measurements are objective and subjective techniques, what is applied in this work is the objective one. The calculation of objective measure was represented by measuring the Peak Signal-to-Noise Ratio (PSNR) and Bit Rate (BR) [13]. PSNR is used to calculate the quality of video sequence as an objective measure and it represents the average value of PSNR at each frame, it's found by the following equation [14]:

$$
P S N R=10 \log _{10} \frac{M A X^{2}}{M S E}
$$

Where the MAX represents the maximum value of the pixel, it's equal to $(2 n-1)$ and the value of $\mathrm{n}$ is the bit number, while the MSE was the Mean Square Error found by the following equation [14]:

$$
M S E=\frac{1}{M N} \sum_{i=0}^{M-1} \sum_{j=0}^{N-1}\left[P_{\text {anchor }}(i, j)-P_{\text {test }}(i, j)\right]^{2}
$$

Where $\mathrm{M}$ and $\mathrm{N}$ represent the picture size with width and height respectively, while the values of $P_{\text {anchor }}$ and $P_{\text {test }}$ are a single-pixel (the first is of the raw picture and the second is of compressed picture) [14].

\section{The Proposed System of Encoding}

The proposed system in this work mainly includes the controller that is incorporated with H.265 codec. This system presents reservation of bandwidth for UHD video streaming especially for widely usage of the Internet, also the number of users changed continuously at the channel that causes a variety of bandwidth availability. Such reasons make the channel status bad at the network traffic congestion. So the controller is used to eliminate this problem and avoid network traffic congestion. The controller is shown in Fig. 2. The goal of this work is the streaming of UHD (4k) video resolution over the Internet. H.265 encoder's features and parameters are used to find the optimal value of BR and PSNR for different levels. The raw video, $4 \mathrm{~K}$ is passed through a subsampling process to produce the other levels, 1080i/p, 720p, 4CIF, CIF and QCIF, see Fig. 2. All levels are encoded with H.265 as a parallel process to make the controller selects the proper level based on the status of the channel buffer. In the steps of this design, each of the levels is worked of optimal PSNR and BR. When the channel suffers from traffic congestion at a time, the controller selects a video sequence with a level less than UHD to be transmitted over the Internet. While at the receiver terminal side, there are two strategies for video reconstruction, if the receiver device is displaying the video with UHD and the received video with a level less than UHD it should be processed by up subsampling or interpolation to retrieve the required level. But if the receiver is displaying different levels with several applications, it is updated to be suitable to that level, which is nearer to the scalability situation [15].

\section{The Proposed System of Video Streaming Over The Internet}

The second part of the proposed system is how to get overall video streaming over the Internet by integrating the encoder operation with the network protocols to examine the network performance and its effects on the video streaming 
quality for different video test sequences. Three classes of video content (high, medium and low motion details) are the video sources to generate an appropriate study about network performance and its parameters, channel bandwidth, packet loss, latency and delay jitter. This mapping of information can be used in the source server to support the QoS to a multimedia distributed delivery system by using the transport and streaming protocols (UDP, RTP, RTCP and RTSP). One basic issue is the network path condition from server to client and impacts on video quality when it suffers from packet loss, latency, bandwidth congestion and delay jitter. This work focuses on how to transmit the video at a low-bit rate taking into account the network status. The multimedia data transmitting over the Internet is very important at the smart devices and applications, so the RTP and its accompanying RTCP have now been developed to distribute the multimedia over internet. This work suggests the use of the RTP to provide the functionality of transforming the real-time data over a network without flow control, while the control is achieved by the RTCP that is used to give feedback about the network path status to allow this system to change the video according to network condition. This work will try to achieve better scalability and performance of video streaming over the Internet with suitable feedback of QoS by this mechanism. Fig. 3 describes the scenario of distributed video with Real-Time streaming over an IP network and the main elements of this experimental set-up.

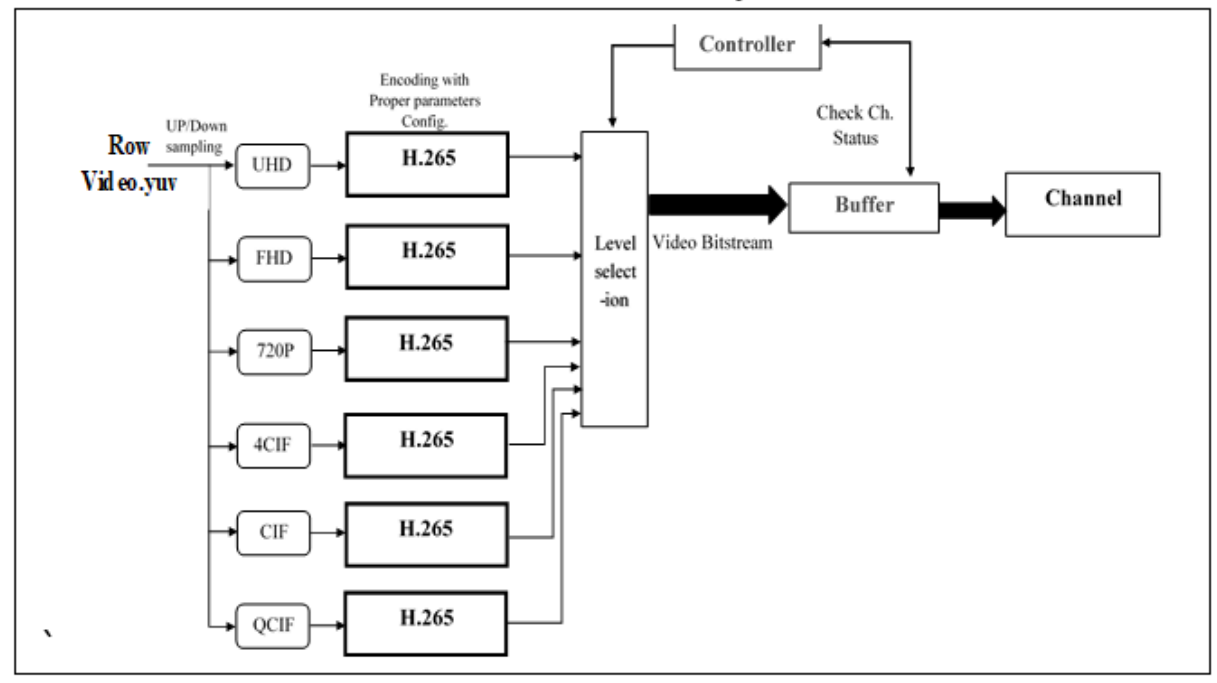

Figure 2: Proposed control system

The test environment contains the source server for video streaming on the Sub-Net1 side and the client at the Sub-Net2 side as shown in Fig. 3. This system uses RTSP for streaming and transmitting by RTP after selecting an appropriate level of video by using RTCP according to network status. While the player requests the streaming content at the server, the server accepts that by RTSP and sends the video streaming to the client using RTP. At the server, there is a network emulator tool used to monitor the network path from server to client and emulate the network parameters conditions. With RTCP, it is used as applied in the standards that give the reports as feedback about QoS with the reflected method that broadcast the SR to all participants in a one-to-many way, while the receiver generates the RR and sends it by the one-to-one way 
not having ability to send with one-to-many. The sender (server) receives the (RR) packet from each receiver (client) in unicast connection (one-to-one way), so the sender is listening to these reports and collecting them and releases to each member by using a multicast group. The (RR) report has the QoS information that goes to the source server to give it the channel status that helps it to select an appropriate level of the encoded video.

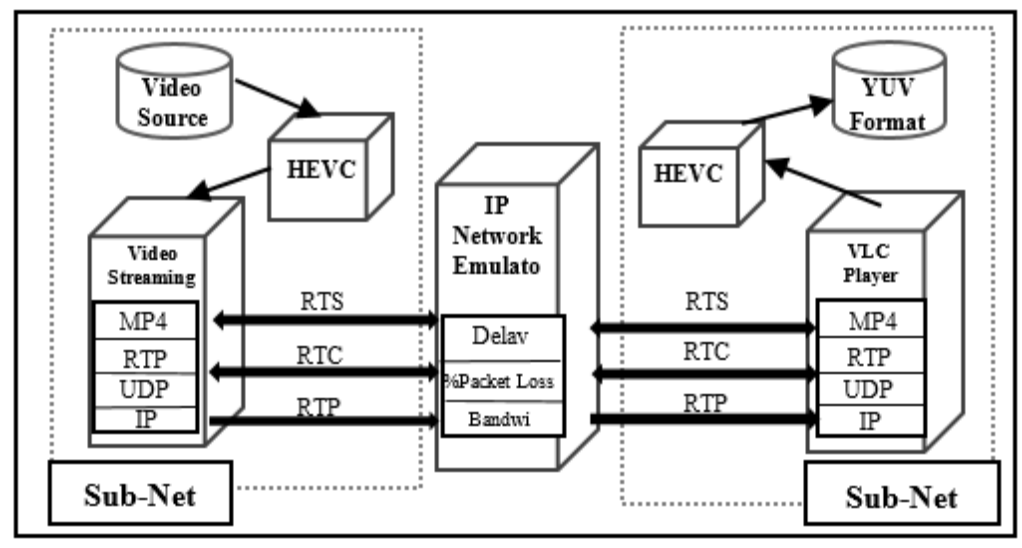

Figure 3: Video streaming network configuration

\section{Work Methodology}

Generally, any recorded video is described by sequential motion activities consisting of different details, low, medium and high. In this experimentation, there are three test sequences (HoneyBee, Jockey and ReadySetGo) with different details and motion, as briefly described in the following.

- The first one is a "HoneyBee" sequence with an object of little movement with a fixed lens, the characteristic of this video is low details.

- The second one is a "Jockey" sequence with the dynamic moving scene and passing the camera to the left, the characteristic of this video is medium details.

- The last one is a "ReadySetGo" sequence with one moving scene and passing the camera to right, the characteristic of this video is high details, see Fig. 4 that describes the first frame of each video.

The proposed work uses six resolutions of these sequences $(4 \mathrm{~K}: 3480 \times 2160$, FHD: $1920 \times 1080$, HD: 720p $1280 \times 720$, 4CIF: $704 \times 576$, CIF: $352 \times 288$, QCIF: $176 \times 144$ ) with 8-bit depth. These three sequences have 100 frames with 120 fps with YUV format (raw data), available in different libraries.

\section{A. Methodology of Encoding Model}

The encoding model that is implemented in this work is described in section 2. Fig. 5 shows the flowchart stages for the proposed model, video source, HEVC/H.265 configuration and controller. 


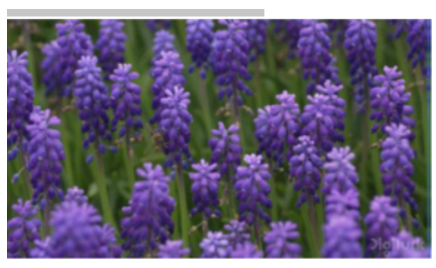

(a)

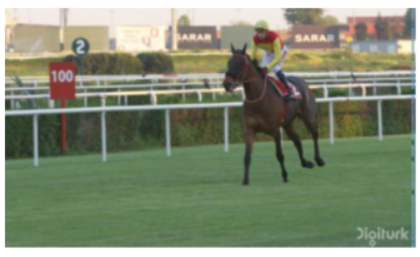

(b)

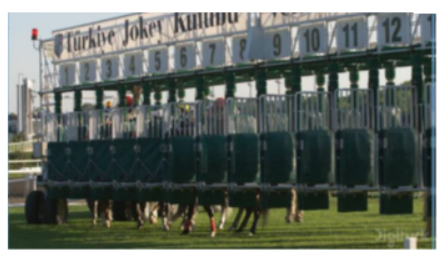

(c)

Figure 4: Three test sequences (a) HoneyBee (b) Jockey (c) ReadySetGo

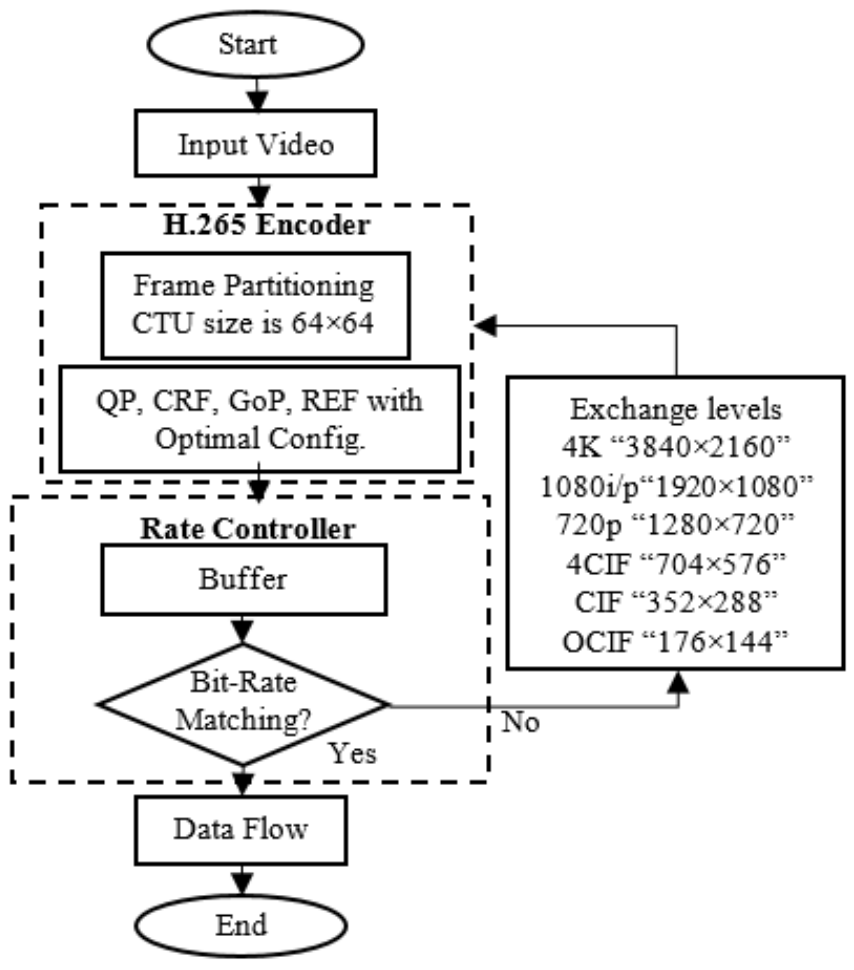

Figure 5: Proposed encoding system flowchart

The available bandwidth of the network channel always changing according to the number of users that share it, this variation needs video bite rate and quality are adapting based on the channel status. The adaptation is achieved by the variation of video resolution applied by the encoder and controller to produce proper video streaming. The configuration 
of H.265 and its features can get the optimal encoded video of each video level. In this work, "ffmpeg" software is used to implement H.265 encoding over three test sequences with changing encoder parameters that directly affect video bit rate and quality. The video quality is represented by the PSNR value. The ffmpeg libraries (libx265 and libavcodec) are used to produce the compression video streaming. The H.265 encoder is applied on raw test sequences with yuv format using coder parameters (QP, CRF, GoP and REF), that are changed to get optimal video bit rate and quality.

\section{B. Methodology of Video Streaming Over The Internet}

The architecture of network streaming as shown in Fig. 3 is implementing by Graphical Network Simulation (GNS3), it can provide a virtual workstation for real network devices. The proposed scenario in this work applies to the network topologies and protocols that now existing. For installing the server device with Windows Server2012 R2, used the virtual machine Qemu template. This server includes one network adapter Ethernet type e1000 interface. Also installing the Client by Qemu VM template with Windows 10. However, the CISCO router with c7200 is installed by Dynamips IOS router template. The structure model of video streaming network content has two scenarios: Unicast and Multicast video streaming. The video is streaming from server to client in a one-to-one way at unicast, while at multicast it's streaming from server to four clients, through the channel (router) that implement in GNS3 as shown in Fig. 6 (a) and (b).

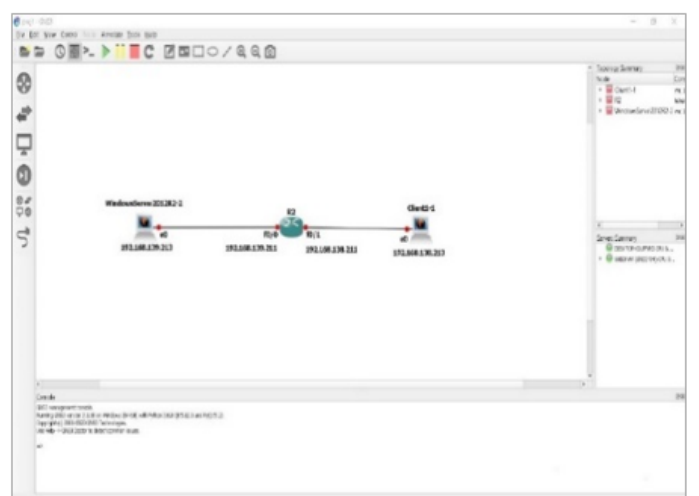

(a)

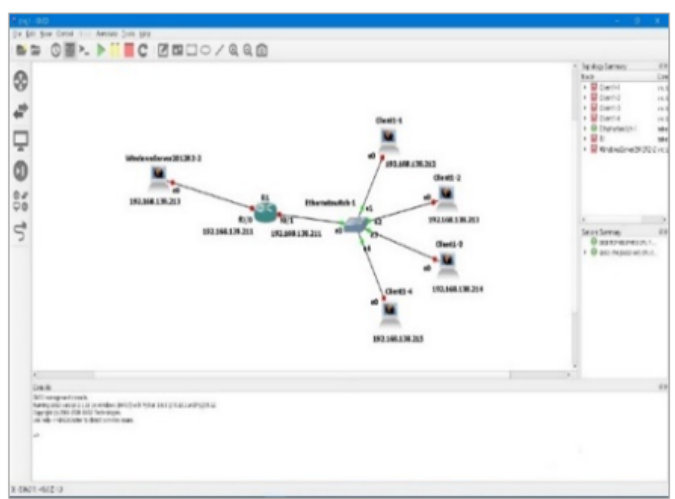

(b)

Figure 6: network video Streaming (a) Unicast and (b) Multicast

At the server, the VLC-player software uses to streaming suitable video sequences, that is prepared with six levels of spatial resolutions. The Cisco router has been configured with suitable IP addresses for two interfaces (Fastethernet $0 / 0$ and 0/1). The interfaces of each device should be set for the unicast and multicast streaming network. Which at the multicasting, the Open Shortest Path First (OSPF) protocol should be configured to achieve reachability. Also, the IGMP group for the multicasting process is implemented on the router interface (f0/1) with IP address (224.4.4.4) by using the command "ip igmp join-group 224.4.4.4". 


\section{EXPERIMENTAL RESUlts}

\section{A. The Experiment Results of H.265 Coder}

From the experiments of three types of test sequences, can conclude the two scenarios:

- First of all, is how to find the optimal configurations of the encoder's parameters with each video layer that gives the required PSNR $(36 \mathrm{~dB})$ as an initial value the system may work with it at the beginning of video compression. While the controller is incorporated with this encoder to achieve the video streaming adaptation on the available bandwidth by selecting an appropriate layer at an assigned bit rate that is compatible with the channel and keeping a good quality for the end-users in the optimal state of the network. From these arguments, one can decide what the optimal configurations of parameters are used, where the value of QP changing according to video's level, will GOP and REF fixed at default values $(\mathrm{GOP}=100$ and $\mathrm{REF}=3)$. This scenario applies to three test sequences but reviews for video with medium details as shown in Table I.

TABLE I

Optimal Configurations Setting for Jockey Video with Six Resolutions

\begin{tabular}{|c|c|c|c|c|c|}
\hline Size & QP & PSNR (dB) & $\begin{array}{c}\text { BR before } \\
\text { H.265 }(\mathrm{Kb} / \mathrm{s})\end{array}$ & $\begin{array}{c}\text { BR after } \\
\text { H.265 }(\mathrm{Kb} / \mathrm{s})\end{array}$ & CR \\
\hline 4K & 46 & 36.921 & 11943936 & 1863.77 & 6408.48 \\
\hline $1080 \mathrm{i} / \mathrm{p}$ & 42 & 36.277 & 2985984 & 1291.53 & 2311.97 \\
\hline $720 \mathrm{p}$ & 40 & 36.003 & 1327104 & 982.95 & 1350.12 \\
\hline 4CIF & 36 & 36.605 & 583925 & 975.9 & 598.34 \\
\hline CIF & 32 & 36.4 & 145981 & 706.9 & 206.50 \\
\hline QCIF & 29 & 36.415 & 36495 & 447.03 & 81.63 \\
\hline
\end{tabular}

- In the second scenario, according to the results from encoded three videos, the critical parameter for determining PSNR is QP. Changing the value of QP to obtain the best range for each layer, that keeps the quality of video acceptable at the values based on the layer type. Table II shows the range of QP used for each resolution. These ranges can be applied to three different motion details at the same video with a long duration. The controller works to check the network condition then return instruction to the encoder to apply an appropriate value of QP based on motion details and layer type. For example, at the 4k layer, the value of QP is (46-48) leading to an accepted range of PSNR (36-33) of encoded video to be transmitted over the channel. While at the smallest resolution (QCIF) can accept the PSNR more than $36 \mathrm{~dB}$.

\section{B. The Experiment Results of Video Streaming}

The evaluation of network performance has two categories: (advanced and simple investigation methodology). The advanced investigation reports the available bandwidth metric of the network path, while the simple investigation reports the packets jitter and delay metrics. These network parameters are evaluated by using the Paessler Router Traffic Grapher (PRTG). The network parameters have a direct effect on video quality. Three encoded videos with six levels for each one are streaming on both the unicast and multicast network, the PRTG sensors should be added to each device and then evaluate the network performance at each case. This evaluation applies by a reflected method when the network uses the RTCP protocol. At this work, the scalability is performed by getting the optimal configuration of the encoder's parameters of each video level. The 077 Ethernet sensor is used to report the results of data that transmit over the unicast network 
Iraqi Journal of Information and Communications Technology(IJICT)

Conference Series: The $1^{\text {st }}$ Conference of Applied Researches in Information Engineering(ARIE2021), 2021

ISSN:2222-758X e-ISSN:2789-7362

path and show how the available bandwidth changes with the different video resolutions, in the same time the ping jitter sensor and the ping sensor are used to monitoring the amount of the delay jitter and the latency respectively. For example, the Fig. 7, 8 and 9) show the results for medium details Jockey video. Fig. 7 describe the (077) Ethernet traffic sensor's results of the advanced investigation, the methodology/bandwidth availability.

TABLE II

Optimal QP Range for Each Resolution

\begin{tabular}{|c|c|c|c|c|c|c|c|c|c|c|c|}
\hline \multirow[b]{2}{*}{ Size } & \multicolumn{3}{|c|}{ HoneyBee } & \multicolumn{3}{|c|}{ Jockey } & \multicolumn{3}{|c|}{ ReadySetGO } & \multicolumn{2}{|r|}{ Rang } \\
\hline & QP & PSNR (dB) & $\overline{B R}$ & QP & PSNR (dB) & BR & QP & PSNR (dB) & BR & QP & PSNR (dB) \\
\hline \multirow{3}{*}{$4 \mathrm{~K}$} & & & & & & & 40 & 36.86 & 7380.61 & 46 & 36.921 \\
\hline & 46 & 36.557 & 792.45 & 46 & 36.921 & 1863.77 & 44 & 35.113 & & & \\
\hline & 48 & 35.74 & & 48 & 35.795 & & 48 & 33.238 & & 48 & 33.238 \\
\hline \multirow{4}{*}{$1080 \mathrm{i} / \mathrm{p}$} & 42 & 36.902 & 453.7 & 42 & 36.277 & 1291.53 & 36 & 36.925 & 5042.38 & 36 & 36.925 \\
\hline & 44 & 35.936 & & 44 & 35.29 & & 40 & 34.888 & & & \\
\hline & 48 & 33.93 & & 48 & 33.257 & & 44 & 32.852 & & & \\
\hline & & & & & & & 48 & 30.858 & & 48 & 30.858 \\
\hline \multirow{4}{*}{$720 p$} & 36 & 38.519 & 446.71 & 36 & 37.989 & 1592.01 & 32 & 37.693 & 4951.52 & 36 & 38.519 \\
\hline & 40 & 36.54 & & 40 & 36.003 & & 34 & 36.638 & & & \\
\hline & 44 & 34.46 & & 44 & 33.966 & & 36 & 35.519 & & & \\
\hline & 48 & 32.336 & & 48 & 31.887 & & 40 & 33.484 & & 48 & 31.887 \\
\hline \multirow{4}{*}{$4 \mathrm{CIF}$} & 32 & 39.233 & 403.84 & 32 & 38.655 & 1591.14 & 28 & 38.689 & 5121.28 & 32 & 39.233 \\
\hline & 36 & 37.019 & & 36 & 36.605 & & 32 & 36.352 & & & \\
\hline & 37 & 36.414 & & 40 & 34.586 & & 36 & 34.182 & & & \\
\hline & 40 & 34.906 & & & & & 40 & 32.143 & & 40 & 32.143 \\
\hline \multirow{4}{*}{ CIF } & & & & & & & 24 & 39.833 & 3417.03 & 24 & 39.833 \\
\hline & 28 & 39.189 & 257.78 & 28 & 38.705 & 1179.43 & 28 & 37.183 & & & \\
\hline & 32 & 36.749 & & 32 & 36.4 & & 29 & 36.551 & & & \\
\hline & 36 & 34.35 & & 36 & 34.231 & & 32 & 34.743 & & 36 & 34.231 \\
\hline \multirow{4}{*}{ QCIF } & & & & 20 & 42.414 & 1309.88 & & & & 20 & 42.414 \\
\hline & 24 & 40.229 & 152.98 & 24 & 39.72 & & 20 & 41.851 & 1952.34 & & \\
\hline & 28 & 37.64 & & 28 & 37.059 & & 24 & 39.013 & & & \\
\hline & 29 & 36.937 & & 29 & 36.415 & & 28 & 36.288 & & 29 & 36.415 \\
\hline
\end{tabular}

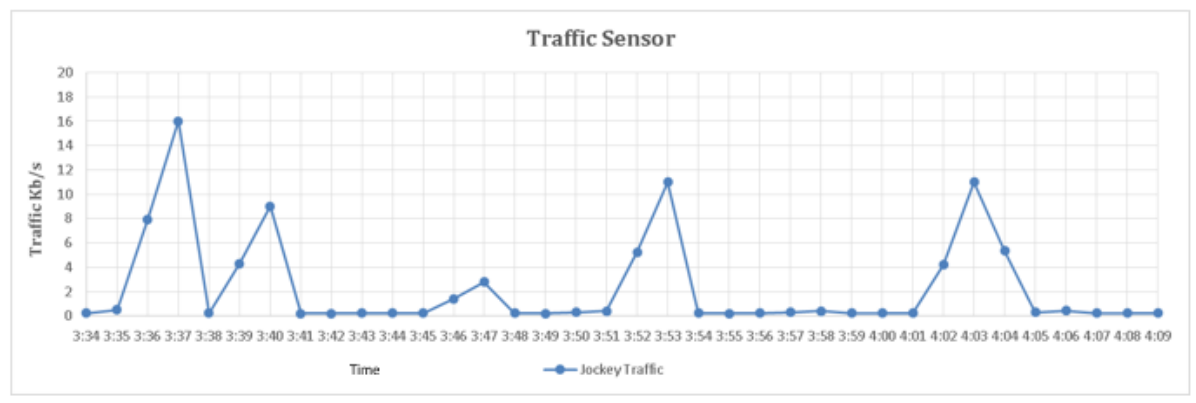

Figure 7: Jockey video traffic

While Fig. 8 describe the ping Jitter sensor's results of simple investigation methodology/delay jitter 


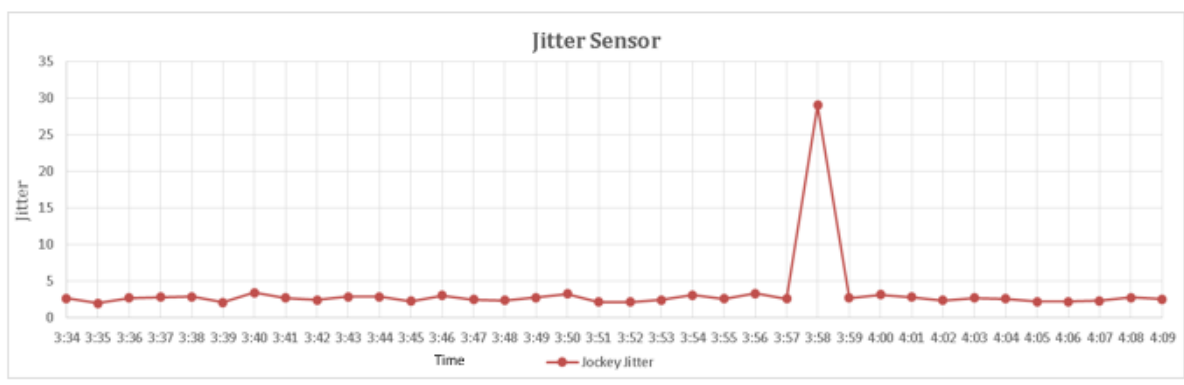

Figure 8: Jockey video delay jitter

Fig. 9 describe the ping sensor's results of simple investigation methodology/delay latency.

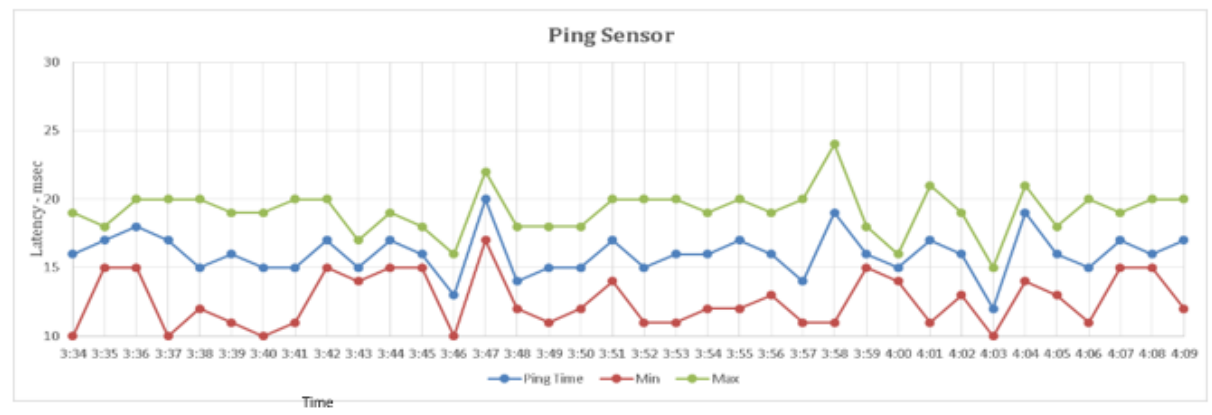

Figure 9: Jockey video delay latency

The main motivation of these experiments is to produce the QoS map of network performance. At the multicasting network, the SNMP sensor uses to report the available bandwidth that is shared among several users as advanced investigation, while the simple methodology evaluates the network performance parameters by PRTG sensors to monitoring the jitter and delay. For example, the Fig. 10, 11, 12 and 13 show the results for medium details Jockey video. Fig. 10 describe the (077) Ethernet traffic sensor's results of advanced investigation methodology/bandwidth availability.

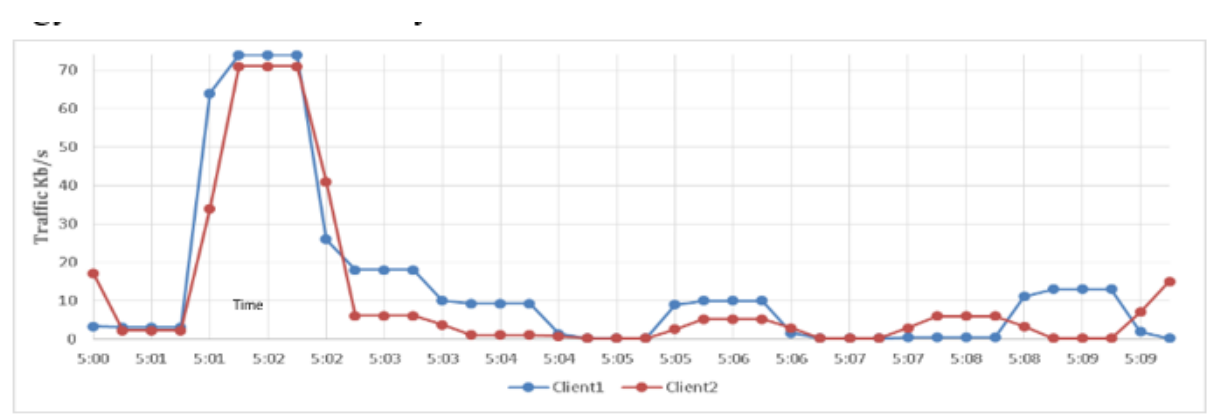

Figure 10: Client (land 2) Jockey video traffics 
While Fig. 11 describe the ping Jitter sensor's results of simple investigation methodology/delay jitter.

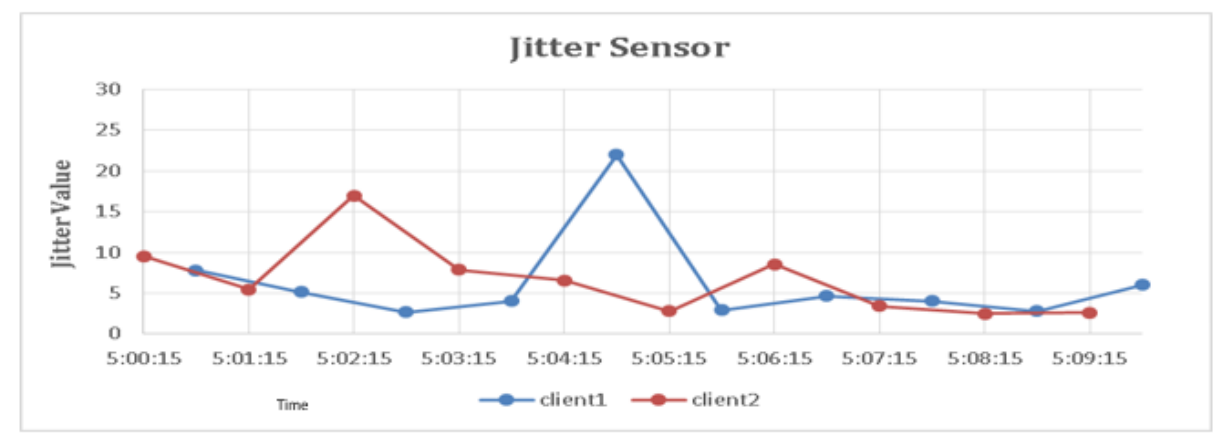

Figure 11: Client (land 2) Jockey video delay jitter

The Fig. 12 and 13 Ping sensor: the results of simple investigation methodology/delay latency.

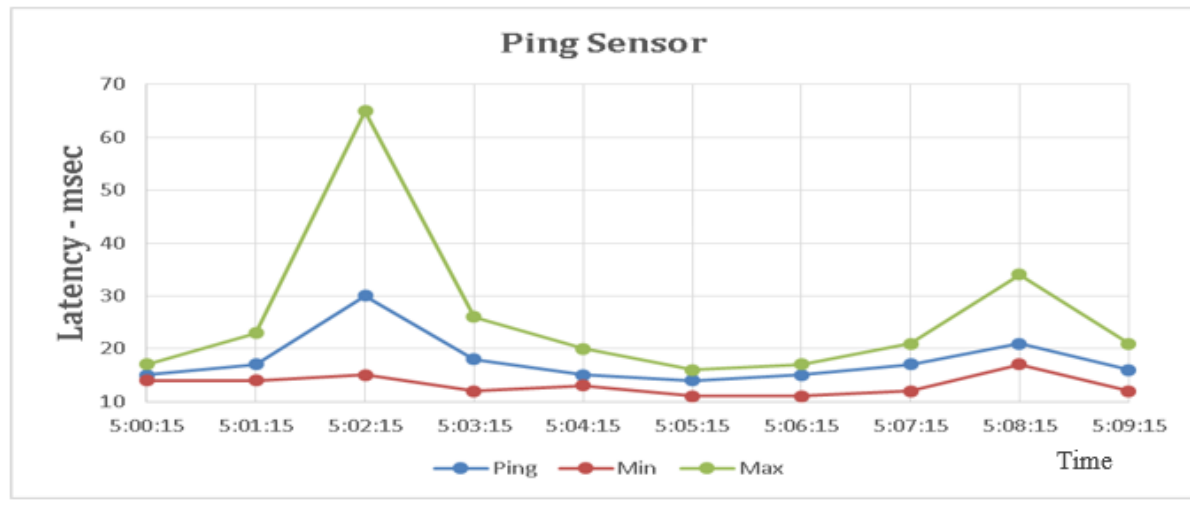

Figure 12: client-1 Jockey video delay latency

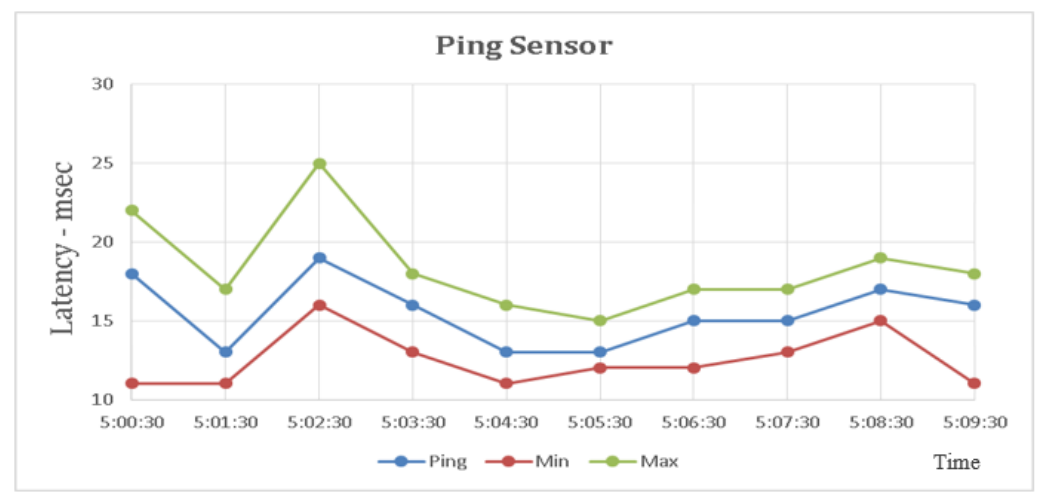

Figure 13: client-2 Jockey video delay latency 
Iraqi Journal of Information and Communications Technology(IJICT)

Conference Series: The $1^{\text {st }}$ Conference of Applied Researches

in Information Engineering(ARIE2021), 2021

ISSN:2222-758X

e-ISSN:2789-7362

\section{Results Discussion}

At the experiments of the first part of this work, the optimal configuration of H.265 parameters should be found at the sender devices with six levels of each test sequence that can be implemented on any video transmitted through the channel. For process time saving of adaptive compression, the critical parameters $(\mathrm{QP})$ of the encoder were applied with four states to finding the best range of acceptable quality (32-40) $\mathrm{dB}$. There are two scenarios of the encoding part, at the beginning the system work with an initial value of PSNR (36) dB, it is achieved by finding the optimal configuration of the encoder's parameters. Then changing the value of QP to get the best range of each video level of resolution as shown in Table II. These ranges can be applied to any video with different motion details and long duration. While at the experiments of the second part of this work, get a global scope of finding the QoS map for the unicast and multicast network performance. At the unicast network, the maximum value of the total traffic for low details video is reached to $10 \mathrm{~Kb} / \mathrm{s}$, while the medium details are reached to $16 \mathrm{~Kb} / \mathrm{s}$ as shown in Fig. 7 , but with the high details is reached to $80 \mathrm{~Kb} / \mathrm{s}$. Also, the range values of the jitter are from 1 to 5 for their videos as shown in Fig. 8. The values of the ping time range $(12-26 \mathrm{msec})$ as shown in Fig. 9, while the maximum latency at the ReadySetGO is $28 \mathrm{msec}$, the ReadySetGo video suffers from delay more than the other two types of videos. At the multicast network, the max value of the traffic achieved to $(25 \mathrm{~Kb} / \mathrm{s})$ for HoneyBee and $(74 \mathrm{~Kb} / \mathrm{s})$ for the Jockey as shown in Fig. 10, while at the ReadySetGo achieved to $(108 \mathrm{~Kb} / \mathrm{s})$. The values of jitter at the four clients range is (2-22) for the HoneyBee and jockey as shown in Fig. 11, while the jitter range at ReadySetGo with high motion details is (2-101) it suffers from jitter more than the other two videos. The delay for this video achieved $195 \mathrm{msec}$ which is more than the other two types of video. The important difference between the results of bandwidth availability, jitter and delay is based on several reasons including the video's content categories that are affected by buffer size, encoding scheme, and video in terms of size, duration, frame rate and bit rate, and finally the user's number at the network.

\section{Conclusion}

In this study, the measurements methodologies of the proposed system of video streaming over the Internet, there are several conclusions. The goal of this study adapted transmitting the UHD video over the Internet according to the channel status and increased the number of users. In this work, there are two topics for the problem solution, video encoding and streaming over the Internet.

- The first topic about encoders:

1) The QP is the most effective parameter, the order of QP adaptation is controller signal based on buffer state.

2) The controller incorporating to choose the suitable format from H.265 multi-layers of sequential resolution.

3) The switching between layers is based on the controller signal buffer state, which achieved scalability.

- The second topic of this work:

https://ijict.edu.iq 
Iraqi Journal of Information and Communications Technology(IJICT)

Conference Series: The $1^{\text {st }}$ Conference of Applied Researches in Information Engineering(ARIE2021), 2021

ISSN:2222-758X e-ISSN:2789-7362

1) The experimental is tested on two networks: unicast and multicast to give an integrated map about the QoS, that applied by streaming three categories of video details on these networks.

2) Each video is monitored with a PRTG monitoring system to get the reports of results to conclude the measurement based on details of the video. From the QoS, the map can be predicted how network conditions affect video streaming over the network.

Furthermore, for optimized the system performance by applying the adaptive compression and adaptive layer selection. The results of the proposed algorithm experiment show the reduction of processing time and improvement of received video quality

\section{REFERENCES}

[1] J.-R. Ohm, G. J. Sullivan, H. Schwarz, T. K. Tan and T. Wiegand, "Comparison of the Coding Efficiency of Video Coding Standards-Including High Efficiency Video Coding (HEVC) ", IEEE Transaction on Circuits and System for Video Technology, Vol. 22, No. 12, Dec., 2012.

[2] M. P. Sharabayko, O. G. Ponomarev and R. I. Chernyak, "Intra Compression Efficiency in VP9 and HEVC", Applied Mathematical Sciences, Vol. 7, No. 137, pp. 6803-6824, 2013.

[3] N. Angelescu, "Objective Video Quality Assessment: Using H264 and H.265 Codecs for 4K Transmissions" , Scientific Bulletin of the Electrical Engineering Faculty, Vol. 1, No. 29, 2015.

[4] L. Anegekuh, L. Sun, E. Jammeh, I. H. Mkwawa and E. Ifeachor, "Content-Based Video Quality Prediction for HEVC Encoded Videos Streamed Over Packet Networks", IEEE Transactions on Multimedia, Vol. 17, No. 8, pp. 1323-1334, Aug. 2015.

[5] S. K. Ibrahim and N. N. Khamiss, "Optimal Usage of LTE Advanced System to Support Multi-User in Video Streaming Application" , IEEE Third Scientific Conference of Electrical Engineering (SCEE), University of Technology, Iraq, 2018.

[6] G. J. Sullivan, J. R. Ohm, W. J. Han and T. Wiegand, "Overview of The High Efficiency Video Coding (HEVC) Standard" , IEEE Transactions on circuits and systems for video technology, Vol. 22, No. 12, pp. 1649-1668, Dec. , 2012.

[7] V. Sze, M. Budagavi and G. J. Sullivan, "High Efficiency Video Coding (HEVC) Algorithms and Architectures", International Publishing Switzerland, Springer, USA, 2014.

[8] M. Majid, M. Owais and S. M. Anwar, "Visual Saliency Based Redundancy Allocation in HEVC Compatible Multiple Description Video Coding" , Multimedia Tools and Applications, Vol. 77, No. 16, pp. 20955-20977, Dec. , 2017.

[9] S. C. Kulasekera, "Multiplierless DFT, DCT Approximations for Multi-Beam RF Aperture and HEVC HD Video Applications: Digital Systems Implementation", M.Sc. Thesis in Science, University of Akron, May, 2016.

[10] Z. Liu, S. Guo and D. Wang, "Binary Classification Based Linear Rate Estimation Model for HEVC RDO" , IEEE International Conference on Image Processing (ICIP), IEEE, 2014.

[11] S. W. Teng, H. M. Hang and Y. F. Chen, "Fast Mode Decision Algorithm for Residual Quadtree Coding in HEVC" , Visual Communications and Image Processing (VCIP), IEEE, 2011.

[12] D. K. Kwon, M. Budagavi and M. Zhou, "Multi-Loop Scalable Video Codec Based on High Efficiency Video Coding (HEVC)" , 2013 IEEE International Conference on Acoustics, Speech and Signal Processing, IEEE, 2013.

[13] S. Tamanna, "Transcoding H. 265/HEVC Video" , Computer Science, Thesis NO. MSE-2013:04, Sweden, Mar. , 2013.

[14] A. Scaccialepre, "Design and Implementation of a Fast HEVC Random Access Video Encoder" , M.Sc. Thesis in KTH electrical engineering, Stockholm, Sweden University, 2014.

[15] H.K. Abd Al-azeez and N.N. Khamiss, "Optimal Quality Ultra High Video Streaming Based H.265" , Indonesian Journal of Electrical Engineering and Computer Science (IJEECS), Vol. 18, No. 3, pp. 1474-1485, June, 2020. 